\title{
A vibrational circular dichroism approach to the determination of the absolute configuration of flexible and transparent molecules: fluorenone ketals of $1, n$-diols
}

\author{
Giovanna Longhi, ${ }^{a b}$ Sergio Abbate, ${ }^{* a b}$ Patrizia Scafato $^{c}$ and Carlo Rosini ${ }^{c}$ \\ Received 3rd July 2009, Accepted 9th February 2010 \\ First published as an Advance Article on the web 17th March 2010 \\ DOI: $10.1039 / b 913213 a$
}

The infrared absorption (IR) and vibrational circular dichroism (VCD) spectra for five ketal molecules, three of which obtained from 1,2-diols and two from 1,3-diols, were recorded in the mid-IR region. The spectra have been satisfactorily reproduced by DFT calculations, even with not too large wavefunction basis sets, especially due to the low number of conformers to be considered. The mobility of some moieties provides a recognizable signature. A characteristic couplet of VCD bands attributed to normal modes involving the methine and a phenyl ring bonded to the stereogenic carbon atom is evidenced for two ketals of the series as a signature of the absolute configuration; due comparison with existing literature is made. A relation is discussed of the present VCD data with the literature VCD data of simple alcohols and diols.

\section{Introduction}

The analysis of the chiroptical properties ${ }^{1}$ (optical rotation, OR, optical rotatory dispersion, ORD, electronic and vibrational circular dicroism, ECD and VCD, respectively, and Raman optical activity, ROA) constitutes a useful tool to assign the molecular absolute configuration (AC). ${ }^{2}$ In addition, the development of quantum chemical methods ${ }^{3}$ as $a b$ initio or density functional theory, DFT, comprehensive of calculations of vibrational properties, and time dependent Hartree Fock, TDHF, or time dependent density functional theory, TDDFT, has allowed the simulation of the above chiroptical properties, making this analysis safer and hence the configurational assignment derived more reliable. All these advancements and the commercial availability of suitable computer packages (Gaussian 03, ${ }^{4}$ Dalton2.0, ${ }^{5}$ TurboMole5.10 6 and more recently $\mathrm{ADF},{ }^{7}$ just to mention those we know of) make, at least in principle, these methods accessible even to the non specialists, as the experimental organic chemists are; after all the latter scientists are those most interested researchers in solving the problem of AC assignment. We would like to point out that, among the theoretical methods, the ones which have shown the best performance in reproducing the experimental data, have been VCD and IR, due to the early definition of the relevant quantities, namely atomic polar tensors (APT) and atomic axial tensors (AAT), and to the development of efficient algorithms for their calculations. ${ }^{8}$ The alternative chiroptical technique related to molecular vibrations, ROA,

${ }^{a}$ Dipartimento di Scienze Biomediche e Biotecnologie, Università di Brescia, Viale Europa 11, 25123 Brescia, Italy.

E-mail:abbate@med.unibs.it

${ }^{b}$ Consorzio Nazionale Interuniversitario per le Scienze Fisiche della Materia (CNISM), Via, della Vasca Navale, 84-00146 Roma, Italy

${ }^{c}$ Dipartiminto di Chimica, Università degli Studi della Basilicata, Via, N. Sauro, 85, 85100 Potenza, Italy is still a challenge for theoreticians, ${ }^{3 a, b, f}$ even though its $a b$ initio interpretation has been around for some time..$^{2 f, 9}$

The problems that still remain, in spite of such significant progress, are mostly related to the treatment of the electronic chiroptical properties of all those compounds showing small $[\alpha]_{D}$ and/or weak or non-existing ECD signals, such as aliphatic alcohols, diols, ethers, amines and so on. In facts, such compounds do not possess typical UV-VIS chromophores and, in addition, they present a large conformational flexibility, so that there occur many conformers with different and even opposite OR values and/or ECD, VCD and ROA spectra, leading to a weighted average value for the latter chiroptical data which may be small. This fact constitutes then the main obstacle to a straightforward analysis of the chiroptical data and then to a reliable AC determination. For a few years, we have been involved in a research project aimed at providing some solutions to this difficult problem. Therefore we have decided to transform the flexible, transparent compounds into chromophoric, conformationally defined derivatives: the analysis of the electronic chiroptical properties of these systems gave simpler and more reliable results, guaranteeing a safe AC assignment. ${ }^{10}$ The same kind of approach was used by Stephens and coworkers ${ }^{11}$ in order to reduce the number of conformers to be considered and thus make the analysis of the VCD spectra of (-)-borneol easier.

In our most recent paper ${ }^{10 i}$ we have shown that by reacting fluorenone with a series of 1,2- and 1,3-diols, the corresponding ketals are obtained in high yield and the conformational flexibility is drastically reduced with respect to the parent molecules. This permits easy and unambiguous determination of the AC of both the derivatised and, as a consequence, of the original molecules, by measuring optical rotation (OR) and electronic circular dichroism (ECD) spectra of the ketals. Due to the reduction in the number of conformers, this system of molecules constitutes an excellent work-bench for testing other methods based on vibrational circular dichroism (VCD). 


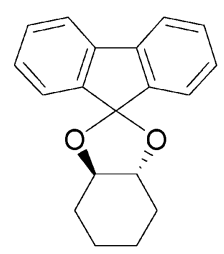

$(4 R, 5 R)-1$

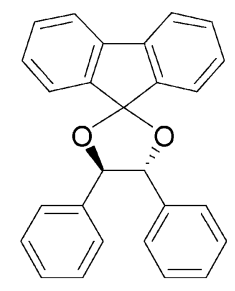

$(4 R, 5 R)-2$

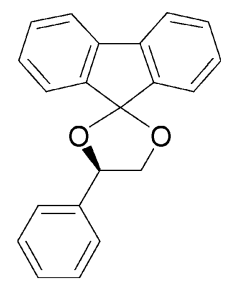

$(R)-3$

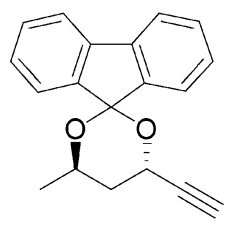

$(4 S, 6 R)-4$

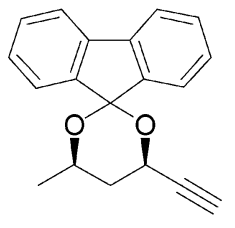

$(4 R, 6 R)-5$
Scheme 1

Indeed the use of VCD for AC determination suffers somewhat from the existence of too many conformers. ${ }^{12,13}$ On the other hand, when the conformers are very few, VCD is a superior technique with respect to other chiroptical spectroscopies. ${ }^{14}$ With this in mind we examine here five ketals from ref. $10 i$, the first three derived from 1,2-diols, and the other two derived from 1,3-diols, namely (+)-(4R,5R)-hexahydrospiro[1,3-benzodioxole-2,9'-fluorene] (1), (+)-(4R,5R)-4,5-diphenylspiro[1,3-dioxolane-2,9'-fluorene] (2), (+)-( $R)$-4-phenylspiro[1,3-dioxolane-2,9'-fluorene] (3), (+)-(4S,6R)-4-ethynyl-6-methylspiro[1,3-dioxane-2,9'-fluorene] (4), and (-)-(4R,6R)-4-ethynyl-6-methylspiro[1,3-dioxane2,9'-fluorene] (5) (see Scheme 1)

The goals of this work are then the following:

(1) To confirm the previously determined configurational assignment of ref. $10 i$;

(2) Especially in relation to point (1), to test whether the DFT calculation of VCD spectra based on the simple choice of B3LYP/6-31G* functional/basis set is enough to yield a good enough prediction of the data or else the more complete (and time-consuming) $\mathrm{B} 3 \mathrm{PW} / \mathrm{TZ} 2 \mathrm{P}$ choice is necessary. ${ }^{15}$

(3) To check whether some VCD spectroscopic features are either associated with different conformers or are influenced by some large amplitude torsional modes, determined either by the puckering of saturated rings or by the hindered rotations of a phenyl groups. This will be studied especially on molecules $\mathbf{3}$ and $\mathbf{5}$.

(4) To see whether the VCD spectra of 1,3-systems are similar or different from those of 1,2-systems. ECD spectra for all these molecules are quite similar, while the OR data are not. ${ }^{10 i}$

(5) We will investigate whether there are some relationships between the ketals VCD and IR spectra and the ones of the parent diols spectra. We will work on this point, using the existing data in the literature: ${ }^{12}$ the present study, based for the rigidification of flexible diols attained by chemical methods, allows in turn us to shed some light also on the VCD data of the parent alcohols.

(6) Finally we will discuss the use of quantitative and objective evaluation methods for judging VCD features, in particular we will examine two recently introduced parameters, i.e. normal mode robustness ${ }^{16}$ and the spectral similarity parameter, the latter one originally defined in the context of ROA spectra analysis. ${ }^{17}$

\section{Materials and methods}

\section{Experimental details}

Synthesis. Compounds 1-5 have been prepared as reported previously. ${ }^{10 i}$

Spectroscopic measurements. IR and VCD spectra were collected on a JASCO FVS4000 FTIR apparatus equipped with a liquid $\mathrm{N}_{2}$-cooled MCT detector, 4000 accumulations were averaged in the $950-1800 \mathrm{~cm}^{-1}$ region at $4 \mathrm{~cm}^{-1}$ resolution. The spectra were obtained for solutions in $\mathrm{CDCl}_{3}$ ranging from $0.15 \mathrm{M}$ to $0.4 \mathrm{M}$ concentrations in $50-100 \mu \mathrm{m}$ pathlength $\mathrm{BaF}_{2}$ cells. The solvent IR and VCD spectra were subtracted from the those of the solutions and the reported results in the ensuing figures correspond to the subtractions.

Computational Methods. Conformational studies have been conducted in ref. $10 i$. Here we paid particular attention to compounds $\mathbf{3}$ and $\mathbf{5}$. The minimum energy structures were optimized by DFT methods with the Gaussian $03^{4}$ package. Two sets of functionals/basis sets were employed: B3LYP/ 6-31G* and B3P91W/TZ2P. The latter basis sets has been constructed as reported earlier, ${ }^{15}$ with information retrieved from the World Wide Web at the URL: www.emsl.pnl.gov/ forms/basisform.html. Harmonic frequencies, dipole strengths and rotational strengths have been calculated, following normal mode analysis and the magnetic field perturbation method to calculate rotational strengths ${ }^{8}$ for conformational energy minima and for some conformations off the minima (vide infra). From these data, IR and VCD spectra were generated by assigning a Lorentzian bandshape to each fundamental vibrational transition, with half width $4 \mathrm{~cm}^{-1}$.

Centerband frequencies were shifted by multiplying by a 0.975 constant factor to facilitate comparison with experimental spectra. The value for parameter is ad hoc and has provided optimal prediction of VCD data in this spectral region; for a thorough discussion of scaling factors see ref. 18. Comparison of experimental and calculated spectra, the latter being generated from frequencies, dipole strengths and rotational strengths contained in the Gaussian 03 output, has been handled through standard software and through Jasco software. Finally the Normal Mode robustness has been calculated by home-made codes. ${ }^{16 c}$

\section{Results and discussion}

We first present and discuss the data for the ketals associated to 1,2-diols (compounds 1, 2, 3) and then, in a separate subsection, those from 1,3-diols (compounds 4 and 5). For each group of molecules we first focus on point (1) and (2) of the introduction and then on points (3), (4) and (5).

The ketals derived from 1,2-diols (compounds 1, 2, and 3)

In Fig. 1, 2 and 3 we compare the experimental IR and VCD spectra with the corresponding calculated data for compounds 


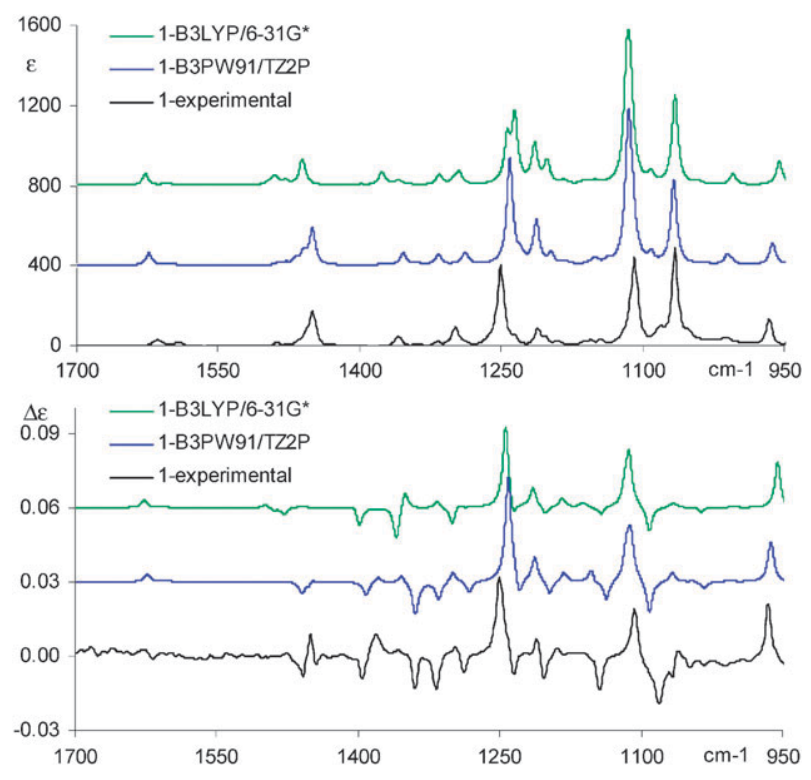

Fig. 1 Comparison of experimental and calculated IR (top panel) and VCD spectra (lower panel) for the 1,2-diol derived ketal 1 in Scheme 1. Spectral resolution for experiment (bottom black trace of both panels) $4 \mathrm{~cm}^{-1}$. Results of DFT calculations based on B3LYP/6-31G* choice (top green trace) and on the B3PW91/TZ2P choice (middle blue trace). Calculated frequencies shifted uniformally by a 0.975 factor. For further details see text.

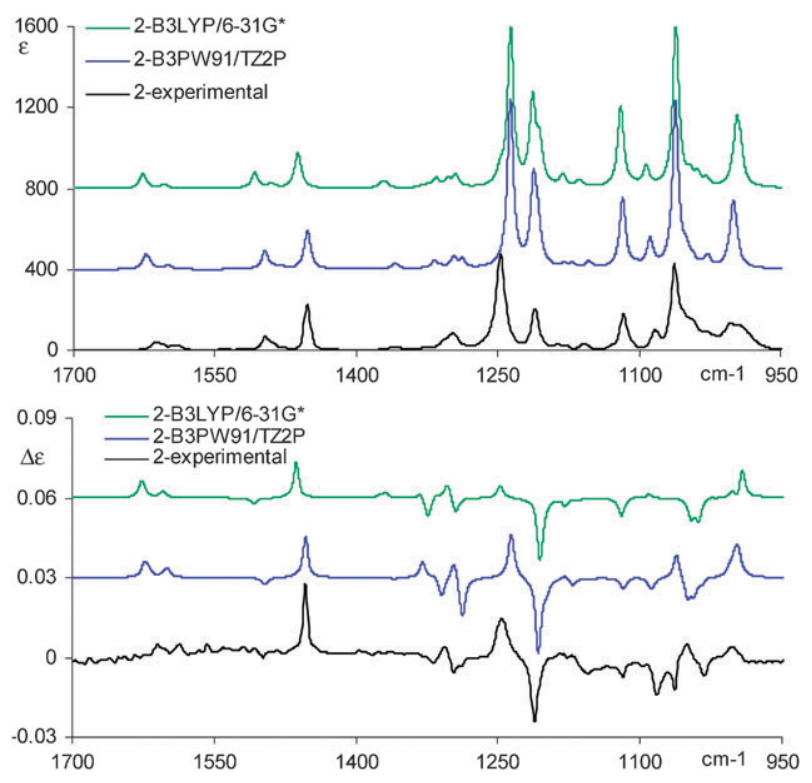

Fig. 2 Comparison of experimental and calculated IR (top panel) and VCD spectra (lower panel) for the 1,2-diol derived ketal 2 in Scheme 1. Spectral resolution for experiment (bottom black trace of both panels) $4 \mathrm{~cm}^{-1}$. Results of DFT calculations based on B3LYP/6-31G* choice (top green trace) and on the B3PW91/TZ2P choice (middle blue trace). Calculated frequencies shifted uniformally by a 0.975 factor. For further details see text.

1, 2 and 3, respectively (for the latter molecule several conformers were tried, vide infra). In all cases we report results both from the $6-31 \mathrm{G}^{*}$ and the TZ2P basis sets: one may immediately verify that the results from both methodologies

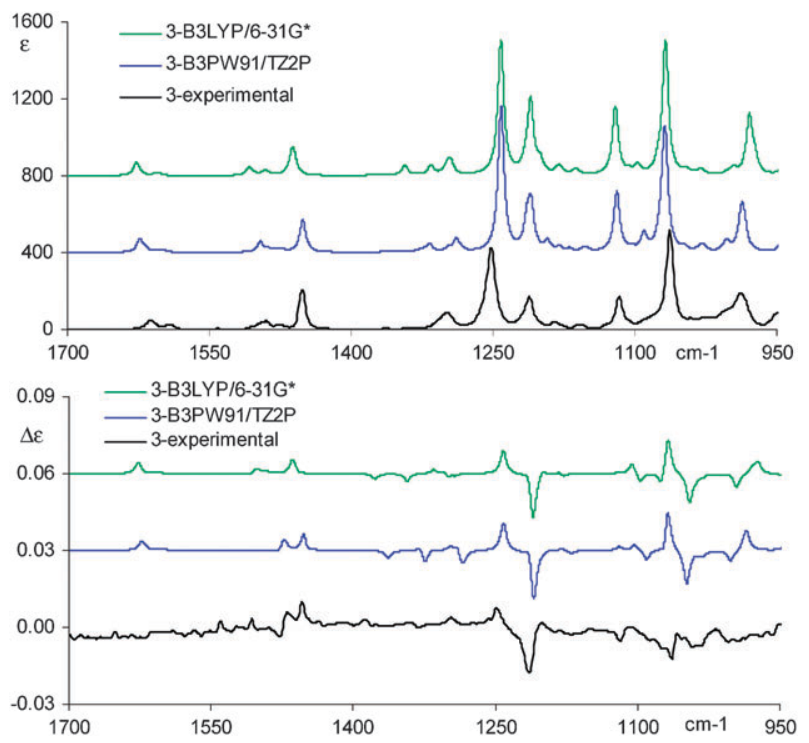

Fig. 3 Comparison of experimental and calculated IR (top panel) and VCD spectra (lower panel) for the 1,2-diol derived ketal 3 in Scheme 1. Spectral resolution for experiment (bottom black trace of both panels) $4 \mathrm{~cm}^{-1}$. Results of DFT calculations based on B3LYP/6-31G* choice (top green trace) and on the B3PW91/TZ2P choice (middle blue trace). Calculated frequencies shifted uniformally by a 0.975 factor. For further details see text.
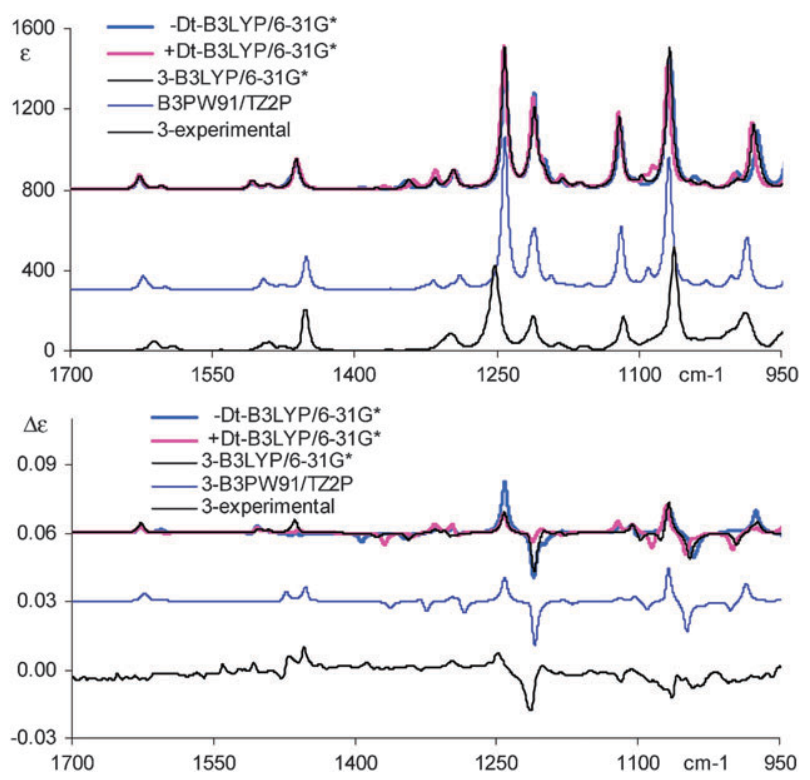

Fig. 4 Comparison of experimental (lower traces) and superimposed calculated IR (top panel) and VCD spectra (lower panel) for the 1,2-diol derived ketal 3 in Scheme 1 for calculated geometries at B3LYP $/ 6-31 \mathrm{G}^{*}$ level at equilibrium and off equilibrium $\left(\Delta \tau=-26^{\circ}\right.$, $-60^{\circ}$, and $+14^{\circ}$ ) (top traces). In the central part of the panels we report also the calculated spectra at B3PW91/TZ2P level for equilibrium geometry. For further details see text and caption of Fig. 3.

are acceptable, notwithstanding the universal judgement that the results obtained with the $6-31 \mathrm{G}^{*}$ basis set are rather poor. Undoubtedly the assignment of AC proposed previously ${ }^{10 i}$ is confirmed here, also based on lower quality calculations. 
We conclude that the computational effort required by employing the TZ2P basis set may not be worthwhile, if the only scope is configurational assignment. Though we recognize that $\mathrm{B} 3 \mathrm{P} 91 \mathrm{~W} / \mathrm{TZ} 2 \mathrm{P}$ provides few finer details with better accuracy especially in the cases of compounds $\mathbf{1}$ and $\mathbf{2}$ : in Fig. 1 the IR band at $1250 \mathrm{~cm}^{-1}$ and the VCD negative pair of bands at $\sim 1300-1400 \mathrm{~cm}^{-1}$ are better predicted in intensity by the high-level calculations. In Fig. 2 we observe that the characteristic intense couplet of alternating signs at $1200-1250 \mathrm{~cm}^{-1}$ is better reproduced in intensity by B3P91W/TZ2P method along with the corresponding IR absorption features. Another VCD couplet at $c a .1050 \mathrm{~cm}^{-1}$ and a negative VCD feature at $c a .1100 \mathrm{~cm}^{-1}$ are better defined by B3PW91/TZ2P calculations.

Considering compound $\mathbf{3}$, we observe that the molecular geometry resulting from optimization is quite similar to that of compound 2, with regards to common molecular moieties. At the B3LYP/6-31G* level, we found also two much less populated conformers within an energy of $2 \mathrm{Kcal} / \mathrm{mol}$, associated with a different conformation of the five membered ring and two phenyl orientations; however these two minor conformers are not stable under B3PW91/TZ2P optimization. In Fig. 3, we present a comparison of the calculated spectra at the two levels B3LYP/6-31G* (lowest energy conformer) and B3PW91/TZ2P. We wish to point out that the main VCD features observed (and calculated) for compounds $\mathbf{2}$ and $\mathbf{3}$ are quite similar indeed: compound $\mathbf{2}$ contains the same VCD active moiety as compound $\mathbf{3}$ sort-of repeated twice, i.e. the same stereogenic carbon bears a phenyl ring with substantially the same geometry (all calculated dihedral angles are very similar). Analogously, we notice that a similar behaviour can be found in a few cases presented in the VCD literature: for example $(2 R)$-butanol and $(2 R, 3 R)$-butanediol, that have very similar mid-IR VCD spectra, with the latter molecule being in practice the former one repeated twice. In fact $(2 R, 3 R)$-butanediol presents three characteristic groups of bands, the first one at $\sim 1072 \mathrm{~cm}^{-1}$ (+ sign), the second one at $\sim 1150 \mathrm{~cm}^{-1}(-)$, the third one at $\sim 1250 \mathrm{~cm}^{-1}(+){ }^{12}$ (Notice that in ref. 13 (2S)-butanol had been studied and the same group of VCD bands was observed with reversed signs). In the present case,
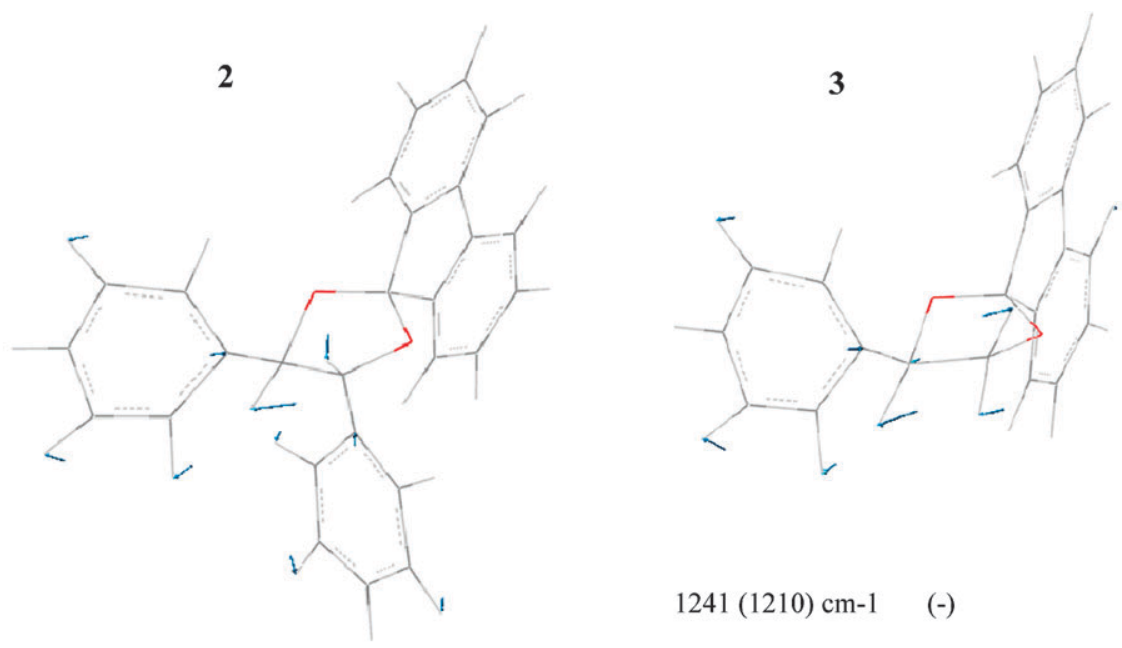

1238 (1207) cm-1

$(-)$

2

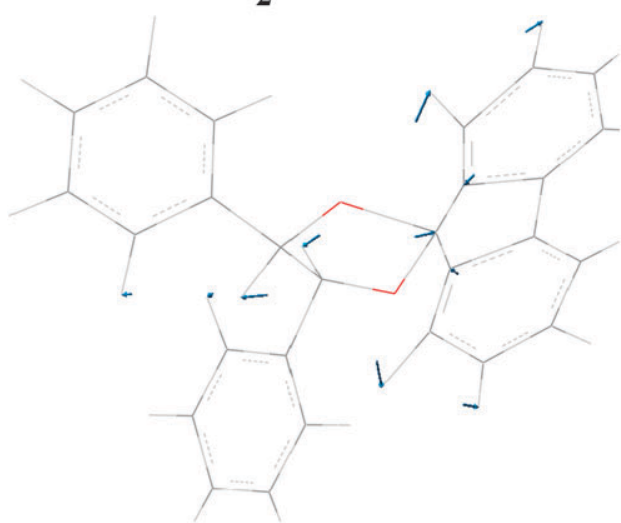

3

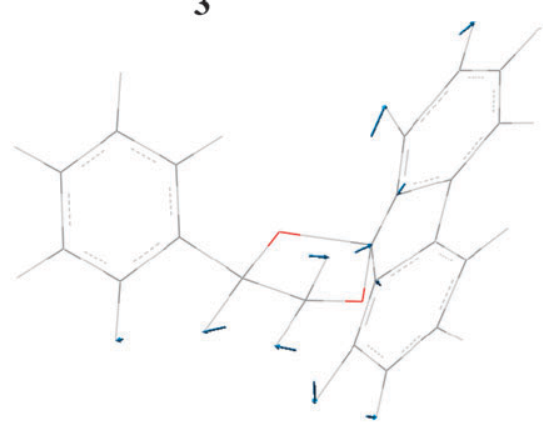

1273 (1241) cm-1

Fig. 5 Calculated atomic displacements for the normal mode eigenvectors providing the most intense VCD (-, +) features for molecules $\mathbf{2}$ and 3 of Scheme 1 in the vicinity of the observed $(-,+)$ couplet between 1270 and $1240 \mathrm{~cm}^{-1}$. In parentheses the scaled values for calculated frequencies are reported. 
where we have a stereogenic center bearing $R$-configuration for $\mathbf{3}$ and a stereogenic $(R, R)$ couple of centers for $\mathbf{2}$, we notice that the group of minor VCD bands between 1000 and $1100 \mathrm{~cm}^{-1}$ are similar and, more importantly, the $(-,+)$ couplet at $1200,1250 \mathrm{~cm}^{-1}$ is similar.

Looking at possible differences between compounds $\mathbf{2}$ and $\mathbf{3}$, one may notice that the presence of just one phenyl ring in compound 3 (with no other bulky group, as e.g. the phenyl close by) may lead to high mobility for the phenyl: in fact the torsion of the phenyl ring has been invoked to account for the observed OR in ref. $10 i$. For this reason we have decided to evaluate if the VCD spectrum of compound $\mathbf{3}$ can be better reproduced, allowing for some hindered rotation or libration of the terminal phenyl ring around the single bond connecting it to the central dioxolane ring. The calculated energy profile for the dihedral angle around such bond was presented in ref. $10 i$ (see the supplementary material therein): it shows low interconversion barriers, close to $3 \mathrm{Kcal} / \mathrm{mol}$; more importantly, it shows a slow increase of energy for torsions $\Delta \tau=\mathrm{OC}^{*} \mathrm{CC}$ around the minimum, which is found at $-26^{\circ}$. If one superimposes the calculated VCD spectra for $\Delta \tau=-26^{\circ}$, $-60^{\circ}$ and $14^{\circ}$ corresponding to the energy minimum and to two values above minimum by about $1 \mathrm{Kcal} / \mathrm{mol}$ in both directions, one has the results of Fig. 4. In this case one may see that the VCD bisignated couplet between 1200 and $1250 \mathrm{~cm}^{-1}$ and the group of bands between 1050 and $1100 \mathrm{~cm}^{-1}$ are quite sensitive to such torsions.

The same phenomenon of VCD sensitivity to phenyl rotational mobility had been observed in $(R)$-naringenin, ${ }^{15}$ especially for the bisignated VCD couplet at $1300-1350 \mathrm{~cm}^{-1}$, that was attributed to modes of the phenyl moiety coupled with the two methine $\mathrm{C} * \mathrm{H}$ deformations mutually orthogonal. Intense VCD couplets associated with vibrations involving the methine and its adjacent phenyl ring had been already reported in other instances in the literature, ${ }^{19,20}$ and we think that this type of vibrations may be taken as diagnostic of the $\mathrm{AC}$ of the stereogenic carbon $\mathrm{C}^{*}$ with a phenyl ring attached to it. We think that at this point it is worth discussing in little more detail the vibrational assignment of the components of the couplet. In particular the negative more intense component of the VCD $(-,+)$ couplet observed here at ca. $1200 \mathrm{~cm}^{-1}$, and calculated at $1241 \mathrm{~cm}^{-1}$ for compound 3 is due to a methine deformation nearly in the phenyl plane, coupled with in plane bendings of phenyl $\mathrm{CHs}$, the positive adjacent band (calculated at $1273 \mathrm{~cm}^{-1}$ for 3 and $1268 \mathrm{~cm}^{-1}$ for 2) being due to the same methine vibration coupled instead with vibrational modes of the dioxolane moiety. For compound $\mathbf{2}$ the methinephenyl deformation giving rise to the VCD negative band is calculated at $1238 \mathrm{~cm}^{-1}$. All this may be better appreciated by looking at Fig. 5, where the normal mode geometries are presented. The same vibration gives rise to a VCD negative band also in the case of $R$-phenyloxirane, as observed by Ashvar et al. ${ }^{19}$ and studied also in ref. 20 and by Jalkanen et al., ${ }^{21}$ for that compound the VCD feature was calculated at $1286 \mathrm{~cm}^{-1}$, while in the case of $(R, R)$-diphenyloxirane it was calculated at $1274 \mathrm{~cm}^{-1}$ (these two modes have been calculated by us at B3PW91/TZ2P level). The sign of the VCD band associated with this particular vibration, which in all the above examples gives the most intense VCD signal in that spectroscopic region, is constantly associated with the $R$-configuration of $\mathrm{C}^{*}$ and may be used as diagnostic of the AC (being the methine and the phenyl the two substituents at the stereogenic carbon of priority 4 and 3 , respectively). The reasons why in $(R)$-naringenin VCD spectra the same couplet is observed $c a .50 \mathrm{~cm}^{-1}$ above what we observe here, namely is at ca. $1350-1300 \mathrm{~cm}^{-1}$, are twofold: in $(R)$-naringenin one has a phenol instead of a phenyl with the $\mathrm{OH}$-bending mode coupling with the methine bending, ${ }^{15}$ and the phenol and methine are close to a six-membered ring bearing a carbonyl.

\section{The ketals derived from 1,3-diols (compounds 4 and 5)}

The comparison of calculated and experimental IR and VCD spectra for compounds $\mathbf{4}$ and $\mathbf{5}$ is provided in Fig. 6 and 7 respectively. Again the results for the B3PW91/TZ2P method are fairly similar (but better) to those obtained with the B3LYP/6-31G* calculation. The VCD spectra of both 4 and 5 are quite different from the ones of $\mathbf{1 , 2}$ and 3: indeed the previously discussed couplet at $c a .1250 \mathrm{~cm}^{-1}$ is present in neither one of the two VCD spectra, since it is characteristic of coupled methine-phenyl vibrations and neither $\mathbf{4}$ nor $\mathbf{5}$ contains this moiety.

One other major fact is that the anti-compound $\mathbf{4}$ has quite a different VCD spectrum from the syn-compound $\mathbf{5}$ not only in the number of VCD active bands, in their signs and frequencies, but especially in the overall intensity, the latter molecule exhibiting a VCD spectrum twice as intense as the former one (on the other hand notice that the IR spectra have similar intensities). This is due to the dioxane ring mobility, which is larger for 4, thus permitting the existence of more conformers, each one with its own VCD spectrum. The calculated results
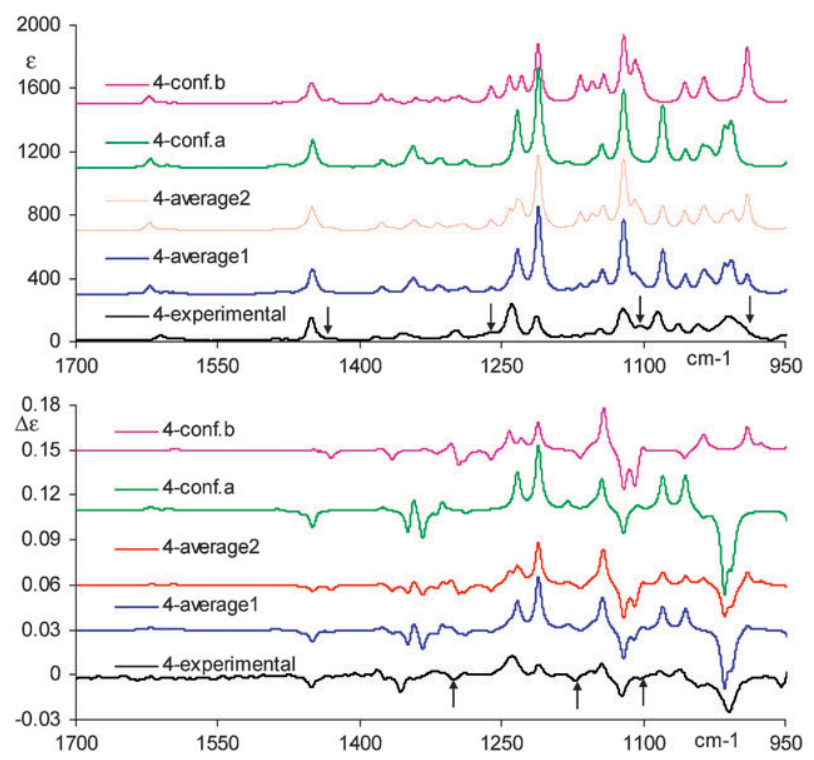

Fig. 6 Comparison of experimental and calculated IR (top panel) and VCD spectra (lower panel) for the 1,3-diol derived ketal 4 in Scheme 1. Spectral resolution for experiment (bottom black trace of both panels) $4 \mathrm{~cm}^{-1}$. Results of DFT calculations based on the B3PW91/TZ2P choice (remaining traces): The VCD and IR spectra of the two major conformers $4 \mathrm{a}$ and $4 \mathrm{~b}$ and their averages according to $\Delta G$ and $\Delta G^{*}$ are reported. Calculated frequencies shifted uniformally by a 0.975 factor. For further details see text. 


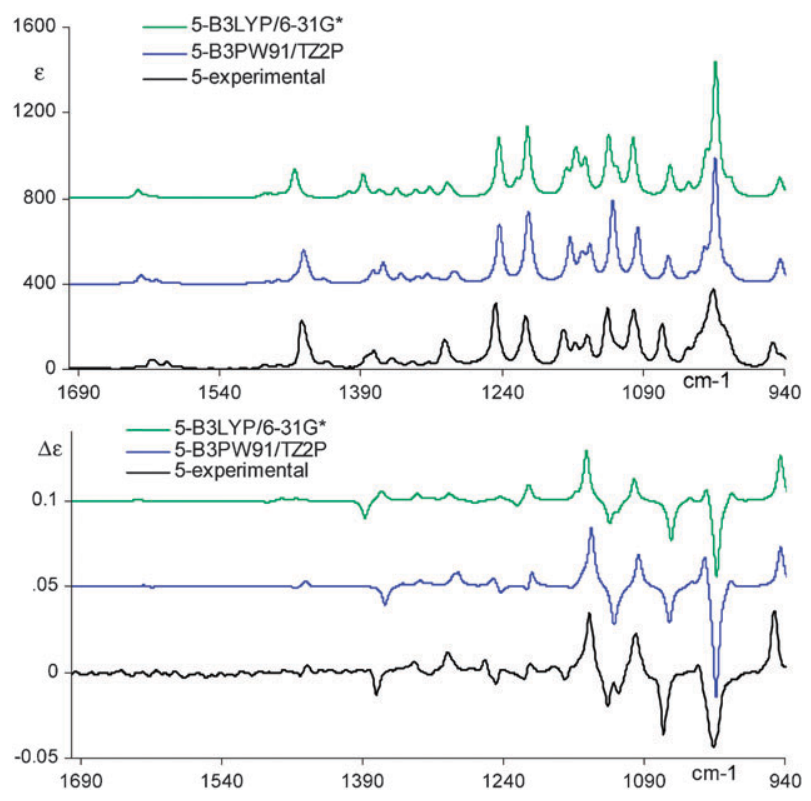

Fig. 7 Comparison of experimental and calculated IR (top panel) and VCD spectra (lower panel) for the 1,3-diol derived ketal 5 in Scheme 1. Spectral resolution for experiment (bottom black trace of both panels) $4 \mathrm{~cm}^{-1}$. Results of DFT calculations based on B3LYP/6-31G* choice (top green trace) and on the B3PW91/TZ2P choice (middle blue trace). Calculated frequencies shifted uniformally by a 0.975 factor. For further details see text.

presented in Fig. 6 for anti-compound 4 refer to the two major conformers reported in Table 1 and to their averages according to different methodologies. Indeed, as usually done in the literature, see for example ref. 22, we have weighed the calculated IR and VCD spectra according to $\Delta G$. From Table 1, one may indeed appreciate that large differences in populations based on $\Delta G$ or $\Delta E$ are predicted for the conformers called $4 \mathrm{a}$ and $4 \mathrm{~b}$. To analyze the origin of such differences and following ref. $23 a$, we have evaluated the quantity $\Delta G^{*}$, for which the thermal corrections to energy and entropy have been calculated by neglecting the low-frequency, large amplitude modes (as described in ref. 23b) (last column of Table 1). The calculated population factors on the basis of $\Delta E$ and $\Delta G^{*}$ are pretty similar and they give, in this case, a poorer result than the calculations based on the usual $\Delta G$ evaluated considering all vibrational modes. Besides the problem of low frequency modes, also the DFT method itself may introduce errors in evaluating conformers population. In any case, as can be judged from Fig. 6, one may conclude that in chloroform the most populated conformer is 4 a since one easily recognizes experimental VCD features that are peculiar of the major conformer $4 \mathrm{a}$ (indeed, notice the pair of positive VCD features below $1100 \mathrm{~cm}^{-1}$ and the negative intense band at $1012 \mathrm{~cm}^{-1}$ ); the low intensity features arising from conformer $4 \mathrm{~b}$ instead are marked with arrows. Still for this analysis the change in the choice of the basis set employed in the calculations is not crucial, as may be gathered from Table 1 .

Another source of differences in the VCD spectra of $\mathbf{4}$ and $\mathbf{5}$, besides conformations, lies in the fact that $\mathbf{4}$ possesses a $(1 S, 3 R)$ stereogenic couple of carbon atoms, whereas $\mathbf{5}$ has a $(1 R, 3 R)$ stereogenic couple of carbon atoms. If we accept the conclusion of the previous section on compounds $\mathbf{2}$ and $\mathbf{3}$, that, both in the diol and in the ketal case, the VCD spectra of compounds with two $R$-centers may be obtained by adding the VCD spectra of compound with one $R$-center, as if the two chiral centers behaved independently of each other and similarly, when the same substituents are at the chiral centers, here we have the following: for compound $\mathbf{5}$ we have a strong VCD spectrum that is due to the sum of two VCD spectra from compounds with two $R$-stereogenic centers, whereas for 4 we have a weaker VCD spectrum originating from a partial subtraction of spectra associated with two opposite configurations.

\section{Conclusions}

In the present work we have studied five ketal molecules by VCD, three of them derived from 1,2-diols and two from

Table 1 Relative energies, Gibbs free energies and population factors of three conformers of compound $\mathbf{4}$ as evaluated with two functional/basis set choices. $\Delta G^{*}$ has been calculated neglecting thermal correction to energy and thermal correction to entropy due to low frequency modes (below $625 \mathrm{~cm}^{-1}$, corresponding to modes excited with minimum $5 \%$ of probability at room temperature). ${ }^{23 b}$ All the dihedral angles of the dioxane ring are reported starting from the one indicated in the scheme and following anticlockwise order (conformer a: methyl and acetyl groups equatorial; conformer b: just methyl equatorial; conformer c: just acetyl equatorial) for the B3PW91/TZ2P calculations

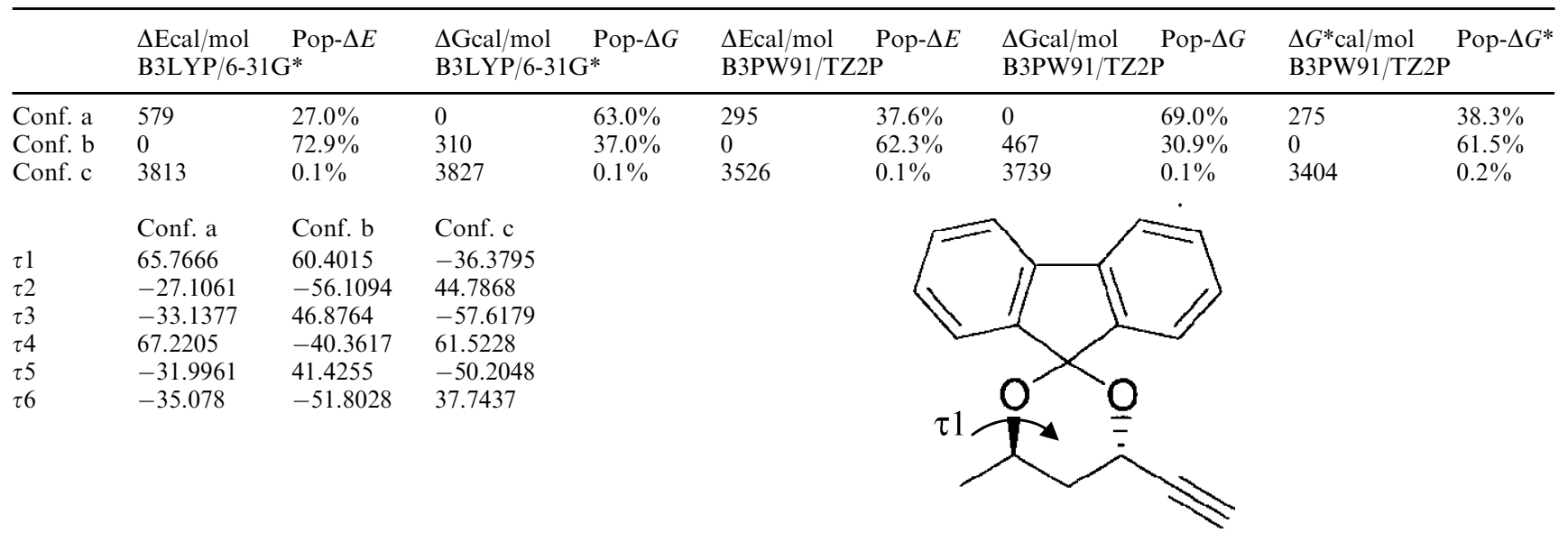


1,3-diols. As stated in the introduction, the study of the OR and ECD data of the ketal molecules studied here has also helped to establish the absolute configuration of the parent diols. On the latter compounds the use of OR and ECD data does not help at all, due to the lack of chromophores. ${ }^{10}$ The chiroptical techniques that do not suffer from the latter limitation are VCD and ROA, which, though, are also difficult to use, when a large number of conformers is present, as in the case of diols. The VCD (and ROA) spectrum in these cases is the sum of the VCD (and ROA) spectra of all conformers; yet the few papers dedicated to the study of the VCD spectra of diols ${ }^{12,13}$ have demonstrated quite specific signals. The present study based on the "rigidification" of the mobile groups, provides a first understanding of the significance of the VCD signals of diols. Following the work plan defined in the introduction, we have achieved the following conclusions:

(1) We have confirmed the previously determined AC assignment derived on the basis of ECD and ORD data, analysed in conjunction with TD-DFT calculations.

(2) To the scope of AC determination, we think that a modest B3LYP/6-31G* method of calculus suffices; however the second choice B3PW91/TZ2P provides surely better predicted spectra and, in the long run, with increased computer power, will be preferred.

(3) When a phenyl is present, one may notice some influence on several VCD bands, most notably on a $(-,+)$ couplet around $(1250,1200) \mathrm{cm}^{-1}$. We have modelled this effect by the calculations presented in Fig. 4 for compound 3, including results also from conformers off equilibrium. More importantly though, we wish to remind the reader that this had been invoked in other instances, to explain broad and intense VCD features. To correctly predict the VCD data, as well as other chiroptical data (ECD and OR), for phenyl containing molecules, it is important to take into account the mobility of the phenyl ring. ${ }^{10 i, 24}$

(4) The VCD spectra of ketals derived from 1,2-diols are in general quite different from the corresponding spectra of ketals derived from 1,3-diols. In general they bear some characteristic signatures from the substituent groups at the chiral centers, as e.g. the methine and the phenyl for molecules 2 and 3. An objective similarity parameter for the experimental VCD spectra of $\mathbf{2}$ and $\mathbf{3}$ may be calculated by integrating over the entire mid-IR region the normalized correlation spectrum $\mathrm{S}_{1}(\nu) * \mathrm{~S}_{2}(\nu)$ (the latter functions referring to the VCD traces of 2 and 3), defined in the last equation of ref. 17b. In this way one obtains 0.5804 , while for the pair of VCD spectra of $\mathbf{4}$ and 5 one obtains 0.4329 . In line with ref. $17 b$, we conclude that both pairs of molecules are similar, with the $\mathbf{2}, \mathbf{3}$ pair being very similar. Besides, they show some relationship to the parent diol molecules. This brings us to consider the next point.

(5) As in the case of parent 1,2-diols or 1,3-diols compared to a simple alcohol, the corresponding ketals with the same groups at the chiral centers show VCD spectral additivity for equal $\mathrm{AC}$ in the two centers or subtractivity for their opposite chirality. We anticipate this effect to be true for 1,4-diols as well as for ketals derived therefrom.

(6) Finally, we wish to comment on the fact that some parts of the VCD spectra may be more important than others for the
AC assignment. Recently Nicu et al. ${ }^{16 a, b}$ introduced the concept of normal mode (NM) robustness, which may help to this purpose. NM Robustness is judged by the angle $\phi$ between the electric dipole transition moment and the
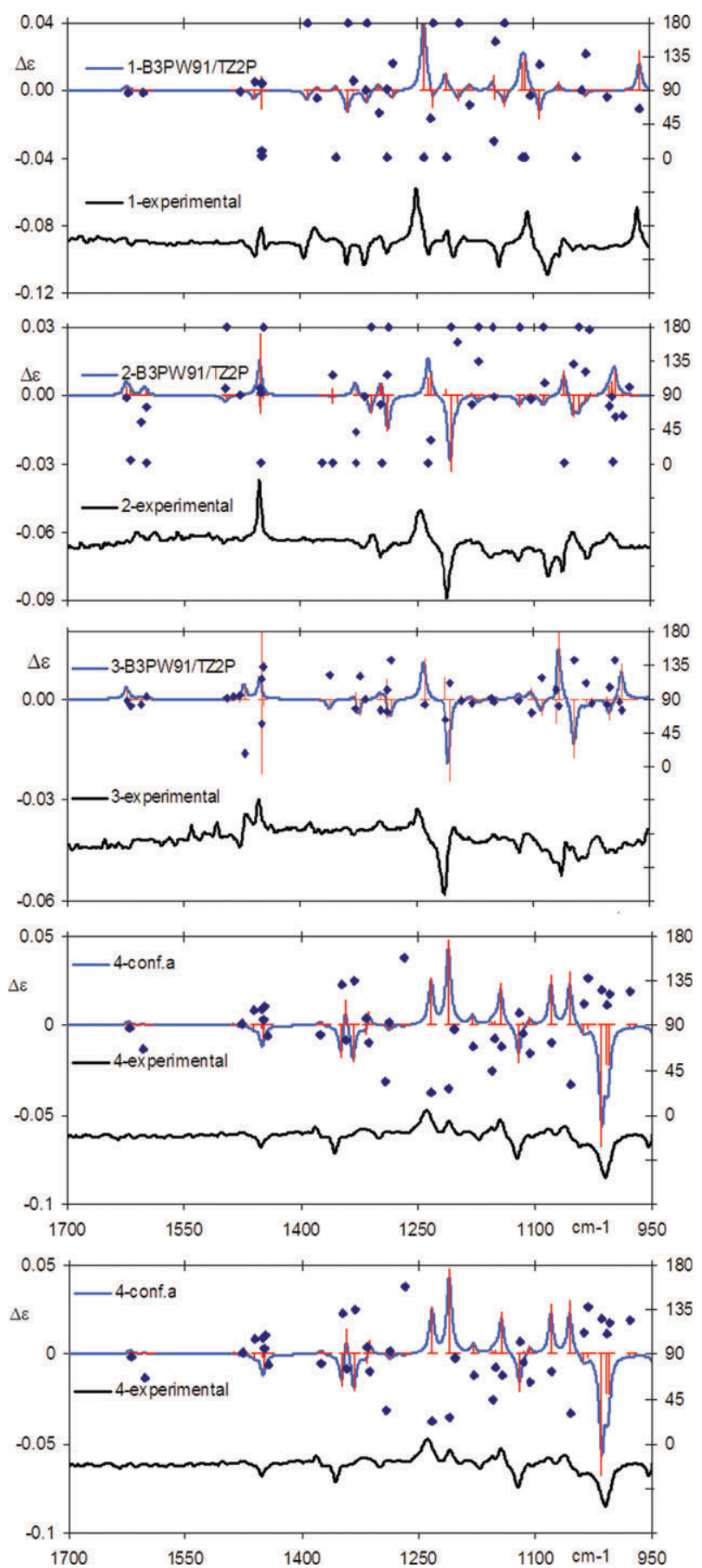

Fig. 8 Experimental and calculated VCD spectra in the mid-IR region for molecules 1-5. of Scheme 1 (top to bottom). Calculated spectra are for the B3PW91/TZ2P functional/basis set. We also provide the robustness parameter $\phi$ value for the intense enough VCD bands (see text). On the left the ordinate scale is for $\Delta \varepsilon$, on the right is for $\phi$. The molecules $\mathbf{1}$ and $\mathbf{2}$ with $C_{2}$-symmetry present many robust modes due to the fact that all $\mathrm{A}$ modes are robust, as noticed in reference. ${ }^{16 b}$ 
magnetic dipole transition moment: a proposed (maybe too strict) criterion is that $\mathrm{NM}$ are considered robust for $\phi$ differing from $90^{\circ}$ for more than $30^{\circ} .{ }^{16 b}$ For this reason we plot in Fig. 8 the experimental and calculated (B3PW91/TZ2P) VCD spectra for all five compounds, together with the $\phi$ values for the most intense VCD features (in ref. $16 b$ VCD features with rotational strengths below a given value are advised to be neglected for fundamental reasons). Limiting ourselves to the $(1240,1210) \mathrm{cm}^{-1}$ couplet for molecules 2 and $\mathbf{3}$, one may see that in $\mathbf{2}$ there are important contributions from robust modes, whereas in $\mathbf{3}$ this is not the case. On this basis, one should not use this $(+,-)$ VCD couplet for safe AC assignment. However, for compound 3 the band at $1210 \mathrm{~cm}^{-1}$ is the dominant feature of the VCD spectrum: direct examination of its NM eigenvector, as presented in Fig. 5, shows that this VCD feature is associated to a methine-phenyl vibration, as previously encountered in other similar molecular systems and recognized as useful for AC assignment.

\section{Acknowledgements}

We thank Dr Lucia Chiummento, Università della Basilicata, for providing some samples for the compounds examined in the present work. We thank also Drs Fabrizio Gangemi and Roberto Gangemi for using their code in evaluating normal mode robustness. Finally we are grateful to the reviewers for useful suggestions.

\section{References}

1 The general theory of chiroptical properties is given by: (a) L. Rosenfeld, Z. Phys., 1928, 52, 161-174; (b) E. U. Condon, Rev. Mod. Phys., 1937, 9, 432-457; (c) A. D. Buckingham, Adv. Chem. Phys., 1967, 12, 107-142.

2 For a general reference, see $(a)$ E. L. Eliel, S. H. Wilen and L. N. Mander, in Stereochemistry of Organic Compounds, Wiley \& Sons, Inc., New York, 1994, ch. 13, pp. 991-1118; use of ECD spectra: (b) N. Harada, in Circular Dichroism: Principles and Applications, ed. K. Nakanishi, N. Berova, R. W. Woody, Wiley-VCH Publishers, New York, 2000, ch. 15, p. 431-458; (c) K. Nakanishi and N. Berova, in Circular Dichroism: Principles and Applications, ed. K. Nakanishi, N. Berova and R. W. Woody, Wiley-VCH Publishers, New York, 2000, ch. 12, p. 337-382; Use of VCD spectra: $(d)$ L. A. Nafie and T. B. N. Freedman, in Circular Dichroism: Principles and Applications, ed. K. Nakanishi, N. Berova and R. W. Woody, Wiley-VCH Publishers, New York, 2000, ch. 4, p. 97-132; (e) P. J. Stephens, in Computational Medicinal Chemistry for Drug Discovery, ed. P. Bultinck, H. de Winter, W. Langenaecker and J. Tollenaere, Dekker, New York, 2003, ch. 26, pp. 699-725; Use of ROA spectra; (f) L. Barron, "Molecular Light Scattering and Optical Activity", Cambridge University Press, Cambridge, UK, 2nd edn, 2004; $(g)$ J. Haesler, I. Schindenholz, E. Riguet, C. G. Bochet and W. Hug, Nature, 2007, 446, 526-529.

3 (a) P. L. Polavarapu, Chirality, 2002, 14, 768-781; (b) M. Pecul and K. Ruud, Adv. Quantum Chem., 2005, 50, 185-227; (c) T. D. Crawford, Theor. Chem. Acc., 2006, 115, 227-245; (d) P. L. Polavarapu, Chem. Rec., 2007, 7, 125-136; (e) T. D. Crawford, M. C. Tam and M. L. Abrams, J. Phys. Chem. A, 2007, 111, 12057-12068; (f) K. Ruud and A. J. Thorvaldsen, Chirality, 2009, 21, E54-E57.
4 M. J. Frisch, et al., GAUSSIAN 03, Gaussian, Inc., Pittsburgh, PA, USA, 2003, http://www.gaussian.com/.

5 T. Helgaker, et al., Dalton, release 2.0, 2005, www.daltonprogram.org. $6 \mathrm{R}$. Ahlrichs, et al., TURBOMOLE, version 5.10, Universität Karlsruhe, 2008.

7 V. P. Nicu, J. Neugebauer, S. K. Wollf and E. J. Baerends, Theor. Chem. Acc., 2008, 119, 245-263.

8 (a) P. J. Stephens, J. Phys. Chem., 1985, 89, 748-751; (b) P. J. Stephens and M. A. Lowe, Annu. Rev. Phys. Chem., 1985, 36, 213; (c) A. D. Buckingham, P. W. Fowler and P. A. Galwas, Chem. Phys., 1987, 112, 1; (d) P. J. Stephens, F. J. Devlin, C. F. Chabalowski and M. J. Frisch, J. Phys. Chem. $A, 1994,98,11623$; (e) J. R. Cheeseman, F. J. Devlin, M. J. Frisch and P. J. Stephens, Chem. Phys. Lett., 1996, 252, 211; (f) P. J. Stephens, C. S. Ashvar, F. J. Devlin, J. R. Cheeseman and M. J. Frisch, Mol. Phys., 1996, 89, 579-594.

9 P. L. Polavarapu, J. Phys. Chem., 1990, 94, 8106-8112.

10 (a) C. Rosini, S. Scamuzzi, G. Uccello-Barretta and P. Salvadori, J. Org. Chem., 1994, 59, 7395-7400; (b) C. Rosini, S. Scamuzzi, M. Pisani Focati and P. Salvadori, J. Org. Chem., 1995, 60, 8289-8293; (c) C. Rosini, G. P. Spada, G. Proni, S. Masiero and S. Scamuzzi, J. Am. Chem. Soc., 1997, 119, 506-512; (d) S. Superchi, M. I. Donnoli and C. Rosini, Org. Lett., 1999, 1, 2093-2096; (e) M. I. Donnoli, P. Scafato, S. Superchi and C. Rosini, Chirality, 2001, 13, 258-265; (f) S. Superchi, D. Casarini, A. Laurita, A. Bavoso and C. Rosini, Angew. Chem., Int. Ed., 2001, 40, 451-454; (g) S. Superchi, D. Casarini, C. Summa and C. Rosini, J. Org. Chem., 2004, 69, 1685-1694; (h) S. Superchi, R. Bisaccia, D. Casarini, A. Laurita and C. Rosini, J. Am. Chem. Soc., 2006, 128, 6893-6902; (i) S. Tartaglia, D. Padula, P. Scafato, L. Chiummiento and C. Rosini, J. Org. Chem., 2008, 73, 4865-4873; (j) S. Tartaglia, F. Pace, P. Scafato and C. Rosini, Org. Lett., 2008, 10, 3421-3424.

11 F. J. Devlin, P. J. Stephens and P. Besse, J. Org. Chem., 2005, 70, 2980-2993.

12 F. Wang and P. L. Polavarapu, J. Phys. Chem. A, 2001, 105, 6991-6997.

13 S. Shin, M. Nakata and Y. Hamada, J. Phys. Chem. A, 2006, 110, 2122-2129.

14 F. J. Devlin and P. J. Stephens, J. Am. Chem. Soc., 1994, 116, 5003-5004.

15 S. Abbate, L. F. Burgi, E. Castiglioni, F. Lebon, G. Longhi, E. Toscano and S. Caccamese, Chirality, 2009, 21, 436-441.

16 (a) V. P. Nicu, J. Neugebauer and E. J. Baerends, J. Phys. Chem. A, 2008, 112, 6978-6991; (b) V. P. Nicu and E. J. Baerends, Phys. Chem. Chem. Phys., 2009, 11, 6107-6118; (c) S. Abbate, F. L. Burgi, F. Gangemi, R. Gangemi, F. Lebon, G. Longhi, V. M. Pultz and D. A. L. Lightner, J. Phys. Chem. A, 2009, 113, 11390-11405.

17 (a) W. Hug and M. Fedorovsky, Theor. Chem. Acc., 2008, 119, 113-131; (b) M. Fedorovsky, H. Gerlach and W. Hug, Helv. Chim. Acta, 2009, 92, 1451-1465.

18 J. P. Merrick, D. Moran and L. Radom, J. Phys. Chem. A, 2007, 111, 11683-11700.

19 C. S. Ashvar, F. J. Devlin and P. J. Stephens, J. Am. Chem. Soc., 1999, 121, 2836

20 F. J. Devlin, P. J. Stephens and O. Bortolini, Tetrahedron: Asymmetry, 2005, 16, 2653-2663.

21 J. Jalkanen, V. W. Jurgensen and I. M. Degtyarenko, $A d v$. Quantum Chem., 2005, 50, 91-124.

22 P. J. Stephens, F. J. Devlin and J. J. Pan, Chirality, 2008, 20, 643-663.

23 (a) H. F. Dos Santos, W. R. Rocha and W. B. De Almeida, Chem. Phys., 2002, 280, 31-42; (b) J. W. Ochterski, in Thermochemistry in GAUSSIAN 03, 2000. Available from http://www.gaussian.com/thermo.htm.

24 E. Giorgio, N. Parrinello, S. Caccamese and C. Rosini, Org. Biomol. Chem., 2004, 2, 3602-3607. 